\title{
Servicios terapéuticos integrados para niños, niñas y jóvenes con necesidades especiales
}

\author{
Integrated therapeutic services for children and young people \\ with special needs
}

\author{
Carlos Vázquez Rivera \\ Pontificia Universidad Católica del Perú \\ prometeos12@hotmail.com
}

\author{
Nancy Berríos Baez ${ }^{2}$ \\ Universidad de Puerto Rico
}

Aracelis Escabí Montalvo 3

Universidad de Puerto Rico

\section{RESUMEN}

El objetivo fue determinar la efectividad de los servicios ofrecidos a niños, niñas y jóvenes con necesidades especiales en las áreas de psicología, terapia ocupacional y del habla-lenguaje en el contexto de un proyecto de base comunitaria. La investigación utilizó el enfoque multimetódico concurrente. Evaluó una muestra de 24 expedientes seleccionados al azar de todas las disciplinas. Se diseñaron dos plantillas (clínica y escolar) para obtener los datos de los expedientes. Entre los resultados se pueden mencionar que, más frecuencia de utilización tuvo la terapia ocupacional con un 79,2 \%, la terapia psicológica con un $54,2 \%$, la terapia de hablalenguaje con un $37,5 \%$ y la de expresión creativa con un $16,7 \%$. Respecto a la participación de los especialistas en las discusiones de casos, el 45,8\% fueron en el área de psicología, el 29,2 \% en terapia ocupacional, el 12,5\% en habla-lenguaje y el $4,2 \%$ en expresión creativa. El estudio alcanzó el $85 \%$ de los objetivos terapéuticos y logró que el 52 \% de las personas participantes aumentaran su desempeño escolar. Algunas conclusiones son: 1) que se cumplieron las metas terapéuticas del proyecto para cada una de las intervenciones; 2) que a pesar de que hubo cambios de terapeutas hubo consistencia en el progreso terapéutico; 3) la necesidad de reevaluar el peso de los aspectos psicosociales como factor que facilita u obstaculiza la terapia $y, 4$ ) confirmamos la necesidad de cambiar de un modelo de prevención a una perspectiva de refortalecimiento para el desarrollo de este tipo de proyectos.

Palabras clave: Evaluación, intervención comunitaria, progreso clínico, factores psicosociales.

\begin{abstract}
The objective was to determine the effectiveness of the offered services to children and youth with special needs in psychology, occupational therapy and speech-language areas in the context of a communitybased project. The research used the concurrent multi-method approach. A sample of 24 randomly selected files from all disciplines was assessed. To gain the data from the files, two templates (clinical and school-related) were designed. The results included that the area most often used was the occupational therapy with $79,2 \%$, the psychological therapy with $54,2 \%$, the speech-language therapy with 37,5 $\%$ and creative expression with 16,7 \%. Regarding to the specialists participation in the discussions of cases, $45,8 \%$ were in the area of psychology, $29,2 \%$ in occupational therapy, $12,5 \%$ in speechlanguage and $4,2 \%$ in creative expression. The study reached $85 \%$ of therapeutic goals and allowed that $52 \%$ of the participants show an increase in their school performance. Some conclusions are: 1) the therapeutic goals of the project for each of the interventions were met; 2) even though some therapists were replaced there was consistency in the therapeutic progress; 3 ) there is a need to reevaluate the weight of the psychosocial aspects as factors that facilitate or hinders therapy, and 4) we confirm the need to change from a prevention model to a perspective of reinvigoration for the development of such projects.
\end{abstract}

Keywords: Assessment, community- based intervention, clinical progress, psychosocial factors.

Historial del artículo:

Recibido: 20 de enero de 2015. Aprobado: 27 de febrero 2015. Disponible en línea: 30 de junio de 2015

1 Doctor en Psicología Social Comunitaria, docente de la Universidad de Puerto Rico en Río Piedras, docente invitado de la Pontificia Universidad Católica del Perú.

2 Magíster en Evaluación Investigativa de la Universidad de Puerto Rico en Ciencias Médicas.

3 Doctora en Psicología Social Comunitaria de la Universidad de Puerto Rico en Río Piedras. 


\section{INTRODUCCIÓN}

Las investigaciones en el campo de la educación especial han demostrado la necesidad de proveer un mayor número de servicios educativos a la población de niños, niñas y jóvenes con necesidades especiales, incluyendo la identificación temprana de sus necesidades. Esto para garantizar y proteger sus derechos y lograr obtener con prontitud las intervenciones educativas y los servicios de ayuda relacionados con sus necesidades especiales. Los estudios sostienen que la intervención temprana propicia un desarrollo integral del niño, la niña y el joven, lo que permite maximizar sus capacidades (1 7).

A partir de dichas investigaciones, nuestra experiencia y el trabajo en el Instituto FILIUS de la Universidad relacionados en las tres áreas mencionadas. Estos estudiantes estaban registrados, o en proceso de ser evaluados, para determinar su elegibilidad al Programa de Educación Especial del Departamento de Educación. El objetivo de esta evaluación investigativa fue determinar la efectividad de los servicios del proyecto a través de una evaluación de resultados. La evaluación de resultados se perfila como una de las herramientas más potentes a la hora de valorar programas de intervención, puesto que proporciona las claves para determinar su mérito y valor a la hora de resolver los problemas para los cuales han sido creados (8). Además, la evaluación se valió de los métodos y criterios de las ciencias sociales con el fin de dotar a sus conclusiones de la mayor precisión y amplitud posibles (9-12).

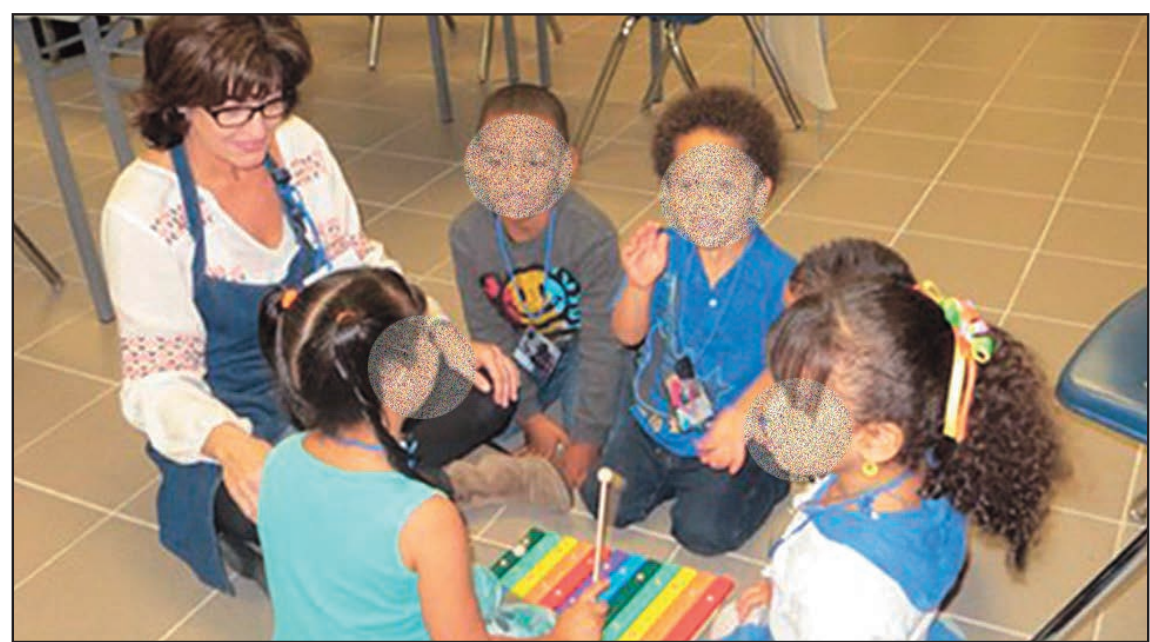

Figura $\mathrm{N}^{\circ}$ 1: Estudio realizado en la ciudad de San Juan por un grupo de investigadores del Programa de Investigación y Refortalecimiento Comunitario, adscrito al Instituto FILIUS de la Universidad de Puerto Rico.

de Puerto Rico, surgió el compromiso de realizar una iniciativa que atendiera de forma más comprehensiva no solo las necesidades, sino también las fortalezas de la población estudiada.

El Programa de Investigación y Refortalecimiento Comunitario (CIReC), adscrito al Instituto FILIUS, realizó una serie de evaluaciones en escuelas públicas en las áreas de psicología, habla-lenguaje y terapia ocupacional a estudiantes que mostraban dificultades en dichas áreas. La experiencia despertó la inquietud y la iniciativa de las personas que integraban $\mathrm{CIReC}$ para ofrecer servicios directos a esta población, impulso que dio origen al denominado "Proyecto de servicios terapéuticos integrados para niños, niñas y jóvenes con necesidades especiales".

El proyecto tenía como meta principal proveer servicios

\section{MATERIAL Y MÉTODOS}

El estudio fue realizado en San Juan, capital de Puerto Rico, entre mayo del 2011 y mayo del 2012. Aplicó la combinación de técnicas cualitativas y cuantitativas. La selección de los expedientes fue aleatoria y se seleccionó un total de 25 expedientes de los 250 disponibles y la selección fue proporcional por género y la cantidad de participantes por escuelas.

Para la evaluación fueron aplicados dos instrumentos desarrollados por el equipo de evaluación de CIReC, tales como la plantilla del expediente clínico y la plantilla del expediente escolar.

La plantilla del expediente clínico fue usada para recopilar información cuantitativa y cualitativa de los expedientes y los datos recopilados con este 
instrumento ofrecieron información para determinar el progreso clínico de las personas participantes. Obtuvo información en torno a seis secciones: a) progreso clínico a partir de los objetivos del análisis del plan de intervención terapéutico (PIT) de las distintas especialidades, b) progreso clínico a partir del análisis de notas de progreso, c) progreso terapéutico, d) factores psicosociales, e) destrezas de las personas participantes en el manejo de su condición y f) adiestramientos a la facultad escolar. Para efectos

Tabla № 1: Diagnósticos por disciplina.

\begin{tabular}{|c|c|c|c|}
\hline \multirow[b]{2}{*}{ Participante } & \multicolumn{3}{|c|}{ Diagnóstico por disciplina } \\
\hline & Psicología & $\begin{array}{c}\text { Terapia } \\
\text { ocupacional }\end{array}$ & $\begin{array}{c}\text { Patología de } \\
\text { habla-lenguaje }\end{array}$ \\
\hline 1 & & Déficit perceptual y déficit visomotor. & \\
\hline 2 & Déficit mental leve. & & $\begin{array}{l}\text { Dificultad severa en lenguaje } \\
\text { receptivo y expresivo. }\end{array}$ \\
\hline 3 & & & $\begin{array}{l}\text { Desorden severo de lenguaje a } \\
\text { nivel expresivo y dificultad severa en } \\
\text { destrezas de conciencia fonológica. }\end{array}$ \\
\hline 4 & & & $\begin{array}{l}\text { Dificultad moderada en lenguaje } \\
\text { receptivo y expresivo, caracterizado } \\
\text { por limitación de vocabulario o } \\
\text { exhibiendo dificultad moderada } \\
\text { en las destrezas de pensamiento y } \\
\text { comprensión auditiva. }\end{array}$ \\
\hline 5 & $\begin{array}{l}\text { Problemas específicos de aprendizaje } \\
\text { (PEA) leve, déficit de atención e } \\
\text { hiperactividad. }\end{array}$ & Déficit perceptual. & $\begin{array}{l}\text { Dificultad en lenguaje receptivo y } \\
\text { expresivo. }\end{array}$ \\
\hline 6 & $\begin{array}{l}\text { Problema moderado a severo de } \\
\text { aprendizaje y de lectoescritura. }\end{array}$ & $\begin{array}{l}\text { Déficit moderado visomotor y déficit } \\
\text { perceptual y coordinación motora. }\end{array}$ & \\
\hline 7 & Déficit de atención (DA). & Déficit visomotor. & \\
\hline 8 & PEA moderado. & $\begin{array}{l}\text { Déficit perceptual visomotor y } \\
\text { coordinación motora. }\end{array}$ & \\
\hline 9 & & $\begin{array}{l}\text { Déficit perceptual e integración } \\
\text { motora. }\end{array}$ & \\
\hline 10 & & $\begin{array}{l}\text { Déficit moderado en el área } \\
\text { visomotora. }\end{array}$ & \\
\hline 11 & PEA en escritura y matemáticas. & $\begin{array}{l}\text { Déficit moderado a severo de } \\
\text { integración motora. }\end{array}$ & \\
\hline 12 & $\begin{array}{l}\text { PEA y problemas en lectoescritura con } \\
\text { déficit de atención e hiperactividad } \\
\text { (DAH). }\end{array}$ & $\begin{array}{l}\text { Déficit moderado perceptual } \\
\text { visomotor. }\end{array}$ & \\
\hline 13 & $\begin{array}{l}\text { PEA y dificultad de lectoescritura. } \\
\text { Indicadores emocionales. }\end{array}$ & $\begin{array}{l}\text { Déficit moderado perceptual y de } \\
\text { coordinación motora. }\end{array}$ & $\begin{array}{l}\text { Rezago en lenguaje receptivo } \\
\text { y expresivo, y destrezas de } \\
\text { lectoescritura. }\end{array}$ \\
\hline 14 & $\begin{array}{l}\text { PEA con rezago en destrezas } \\
\text { visoperceptuales y problema en } \\
\text { manejo de emociones. }\end{array}$ & $\begin{array}{l}\text { Déficit moderado a severo perceptual } \\
\text { visomotor y coordinación de motor } \\
\text { fino. }\end{array}$ & \\
\hline 16 & PEA lectoescritura. & Déficit perceptual visomotor. & \\
\hline 17 & $\begin{array}{l}\text { DAH y PEA, dificultad en lectoescritura } \\
\text { y baja autoestima. }\end{array}$ & Déficit perceptual visomotor severo. & \\
\hline 18 & $\begin{array}{l}\text { Dificultad en integración sensorial y } \\
\text { problema de conducta. }\end{array}$ & Funcionamiento adaptativo bajo. & $\begin{array}{l}\text { Déficit severo en lenguaje receptivo } \\
\text { y expresivo; déficit en integración } \\
\text { sensorial habla - lenguaje; } \\
\text { regulación de conducta. }\end{array}$ \\
\hline 19 & & Dificultad leve perceptual y visomotor. & $\begin{array}{l}\text { Déficit moderado lenguaje } \\
\text { expresivo; déficit moderado en } \\
\text { habla y articulación. }\end{array}$ \\
\hline 20 & & Déficit perceptual visomotor. & Rezago leve en articulación. \\
\hline 21 & & Déficit perceptual visomotor. & \\
\hline 22 & & Déficit perceptual visomotor. & \\
\hline 23 & & Déficit leve perceptual y visomotor. & \\
\hline 24 & & $\begin{array}{l}\text { Déficit perceptual y de integración } \\
\text { visomotora. }\end{array}$ & $\begin{array}{l}\text { Rezago moderado en lenguaje } \\
\text { receptivo y expresivo; dificultad } \\
\text { moderada en habla. }\end{array}$ \\
\hline
\end{tabular}


de este artículo solo se incluyó la información de las primeras cuatro secciones.

La plantilla del expediente escolar sirvió para recopilar información del expediente académico de las personas participantes. Comprendió tres secciones constituidas por un total de 13 preguntas. Estas secciones permitieron obtener: a) información general de la persona participante, b) aprovechamiento académico y c) conducta.

Para cumplir con el procedimiento, adiestramos a nuestro equipo de evaluación en el uso de ambas plantillas y asignamos un total de cinco expedientes a cada una de las personas colaboradoras. La revisión de cada expediente consistía en examinar los siguientes documentos: a) certificaciones de servicios, b) informes de evaluación, c) historial de desarrollo, d) entrevistas de psicología, terapia ocupacional y hablalenguaje, e) minutas de discusión de caso, g) plan de intervención terapéutico y h) notas de progreso. Con esta recopilación se construyó un banco de datos para ingresar la información.

También solicitamos permiso a la dirección de las escuelas para acceder a los expedientes escolares de las personas participantes. Recibida la autorización, el equipo de evaluación se movilizó a las escuelas en las fechas acordadas y examinaron los expedientes académicos de los participantes seleccionados. EI equipo examinó la tarjeta acumulativa y el registro de notas correspondientes a los años de evaluación de los expedientes y documentaron la información.

Ambas planillas produjeron datos cuantitativos y cualitativos. Para los datos cuantitativos se aplicaron estadísticas descriptivas y fueron analizados mediante el programa estadístico SPSS y Excel.

Por otro lado, desarrollamos análisis de contenido de las verbalizaciones relacionadas con el progreso clínico en las distintas terapias. Realizamos una lectura inicial de las notas de progreso para identificar las categorías que describían aquellos factores que pudieron incidir en el progreso clínico y luego una segunda lectura para confirmar las categorías desarrolladas.

\section{RESULTADOS}

\section{Género y edad}

De la muestra de 25 expedientes, se evaluó $24 ; 16$ $(66,7 \%)$ fueron del género masculino y $8(33,3 \%)$ del género femenino.

En el $50 \%$ de los expedientes analizados encontramos que las personas participantes tenían entre cinco y siete años de edad cuando comenzaron en nuestro proyecto, mientras que el $83,4 \%$ entre ocho y doce años al momento de la evaluación de los expedientes.

Según el tipo de terapias que se ofrecieron durante el período de evaluación, la terapia con más frecuencia de utilización fue la terapia ocupacional, total 19 $(79,2 \%)$; seguida por la terapia psicológica, 13 (54,2 $\%)$; la terapia de habla-lenguaje, 9 (37,5\%); y la de expresión creativa con $4(16,7 \%)$.

En lo que concierne a la participación de las especialistas en las discusiones de casos, los expedientes reflejaron que 11 (45,8\%) fueron en el área de psicología, 7 $(29,2 \%)$ en terapia ocupacional, $3(12,5 \%)$ en hablalenguaje y $1(4,2 \%)$ en expresión creativa. Debemos considerar que las discusiones de casos son actividades desarrolladas para atender la preocupación del padre, madre, o persona encargada, algún miembro de la facultad, o de trabajo social, que al notar en el niño o niña algún factor perjudicial relacionado con su educación o comportamiento, solicita para que se discuta de manera interdisciplinaria.

\section{Diagnóstico}

En la tabla $\mathrm{N}^{\circ} 1$ mostramos una lista con los diagnósticos por disciplina, al momento de la evaluación de los expedientes. Como observamos, en algunos casos las personas participantes recibieron dos o más diagnósticos en el mismo período de servicios.

\section{Progreso por objetivos terapeúticos}

El progreso terapéutico representa la relación que se desarrolla entre el terapeuta y la persona participante. La relación terapéutica es la utilización de los atributos del terapeuta de manera artística y selectiva con el fin de posibilitar la terapia (13). Este autor también menciona que surge para generar un vínculo imprescindible para la relación de ayuda con los pacientes. Arango (14) concluye que la relación terapéutica es una construcción social que se transforma constantemente en el quehacer del terapeuta, que está ubicada en un contexto histórico y social y que responde a las necesidades y condiciones del momento.

De acuerdo con la frecuencia y los porcentajes relacionados con los indicadores de progreso terapéutico, las personas participantes se mantuvieron con más frecuencia en terapia ocupacional después de transcurridos dos meses, pero también fue en esta área en la que hubo más resistencia a las recomendaciones de las terapeutas y a asistir a las terapias. Sin embargo, en habla-lenguaje hubo una reducción en los patrones de ausentismo de dichos participantes.

En general hubo un $25 \%$ de ausentismo a las terapias como se desprende de la tabla $\mathrm{N}^{\circ} 2$. Aunque el patrón de ausentismo en todos los tipos de terapia fue 
Tabla $N^{\circ}$ 2: Porcentajes de ausencias a terapias por disciplina.

\begin{tabular}{lcrr}
\hline \multicolumn{1}{c}{ Terapia } & $\begin{array}{c}\text { Cantidad de } \\
\text { terapias }\end{array}$ & Ausencias \\
\hline Patología de habla-lenguaje & 297 & 98 & 33 \\
Terapia de expresión creativa & 101 & 26 & 26 \\
Terapia ocupacional & 908 & 223 & 58 \\
Psicología & 317 & 405 & 25 \\
Total & 1623 & 25 \\
\hline
\end{tabular}

básicamente el mismo, en habla-lenguaje fue un poco más elevado, mientras que en psicología fue un poco más bajo.

Los resultados reflejan que el 78,9\% (15/19) de las personas participantes que tomaban terapia ocupacional evidenciaron algún progreso, también ocurrió lo mismo con los participantes de otras terapias, con el $76,9 \%(10 / 13)$ que participaban en psicología, con el 50,0\% (2/4) en expresión creativa y con el 33,3 \% (3/9) en habla-lenguaje.

Podemos concluir que virtualmente en el $100 \%$ de los casos analizados hubo progreso terapéutico a pesar de los cambios de terapeutas, interrupciones en los servicios, los recesos usuales en las escuelas, la dilatación en las contrataciones de las terapeutas y algunos conflictos propios de las escuelas.

\section{Progreso clínico}

Para efectos de esta evaluación se utilizaron los criterios de progreso clínico establecidos en el Departamento de Educación que se describen de la siguiente forma para alcanzar los objetivos terapéuticos: L1: muestra interés y lo intenta; L2: lo logra con mucho apoyo, dirección y uso de claves; L3: lo logra con algún apoyo, dirección y uso de claves; L4: lo logra por sí mismo en forma inconsistente, y L5: lo logra en forma independiente y consistente.

Evaluamos un total de 404 objetivos documentados en los planes de intervención terapéutico en psicología, terapia ocupacional y patología de habla-lenguaje. Del total de los objetivos evaluados hubo progreso en el 345 (85\%).

17 (59\%) de los objetivos en psicología alcanzaron un progreso clínico de L4 o L5; 120 (40\%) de los objetivos de terapia ocupacional muestran un progreso hacia L3, seguido muy de cerca con el 114 (38 \%) objetivos documentados con un progreso hacia L4; y en cuanto al progreso clínico informado para habla-lenguaje, el nivel alcanzado fue hacia L5 para 29 (39\%) de sus objetivos.

\section{Factores psicosociales}

Aunque el concepto psicosocial es amplio y de difícil definición, para esta investigación consideramos factores psicosociales a las manifestaciones interpersonales del comportamiento que conllevan interacciones y múltiples influencias y que tienen en consideración los contextos sociodemográficos, socioculturales y sociopolíticos en las sociedades contemporáneas $(15,16,17)$.

Los primeros tres factores protectivos del contexto de tratamiento fueron la presencia de figuras maternas $(95,8 \%)$ y paternas $(70,8 \%)$ en el hogar, conjuntamente con haber recibido los servicios correspondientes a sus necesidades especiales por parte de la escuela $(66,7 \%)$. Los primeros tres factores no protectivos, en cambio, fueron la dificultad en las áreas de lectura $83,3 \%$ ) y escritura $(70,2 \%)$, y la ausencia del padre en el hogar (58,3\%).

\section{Progreso escolar}

El promedio académico fue el indicador que seleccionamos para evaluar el progreso escolar de las personas participantes. Comparamos los promedios escolares de 21 de los 24 expedientes y encontramos que 11 (52 \%) mostró un aumento en sus promedios desde que comenzaron las terapias, mientras que el restante 10 (48\%) mantuvo sus calificaciones de inicio de terapia. De los 11 que mostraron aumento en sus promedios, 9 (82 \%) de los niños y niñas aumentaron al menos una nota (p.e. de $\mathrm{D}$ a $\mathrm{C}$ o de $\mathrm{B}$ a $\mathrm{A}$ ) desde las terapias y 2 (18\%) aumentó dos o más notas (p.e. de $C$ a $A$ o de D a B). En muchos casos estos cambios en las notas también responden a una mejor comprensión de los padres, madres, persona encargada y/o el personal escolar de la condición de los niños, niñas y jóvenes en los distintos contextos donde interactúan.

\section{Análisis cualitativo}

Los resultados del análisis cualitativo provienen de la información obtenida de los expedientes, relacionada con las notas de progreso. Estos resultados fueron establecidos en función de categorías, subcategorías y las respectivas verbalizaciones. Las categorías fueron progreso clínico (con sus subcategorías: psicología, 
terapia ocupacional, habla-lenguaje), factores psicosociales, técnicas utilizadas en terapia y fortalezas del niño o niña.

\section{Progreso clínico}

Para determinar el progreso clínico en esta muestra se consideraron los objetivos a trabajarse en el plan de intervención terapéutico (PIT), la severidad de los diagnósticos y los factores psicosociales presentes en la vida de los niños y niñas. En un $85 \%$ de los expedientes analizados se evidenció progreso clínico, por lo que decidimos hacer un doble cotejo y realizar un análisis cualitativo de los expedientes.

En la terapia de psicología, las áreas más notables en que algunos de los niños y niñas mostraron progreso fueron: a) identificación de sus emociones, b) expresión y manejo de emociones, c) autoestima y d) confianza en sí mismo. En términos generales, algunos de estos niños y niñas no podían identificar sus emociones ni aspectos positivos de sí mismos y en otros, las terapias tuvieron un impacto directo en el progreso escolar y en las interacciones sociales con sus pares y familiares.

También se trabajaron las áreas de atención, concentración, tolerancia a la tarea y planificación con algunas personas participantes. Dentro de estas áreas encontramos niños y niñas que trabajan desde un nivel de L2 a L5.

En aquellos casos más severos de rezago en áreas de comunicación e interacción social, observamos un progreso de L1 a L2. A pesar de que no es el máximo nivel de progreso, representa una posibilidad para trabajar otras áreas necesarias y así alcanzar los objetivos y las metas terapéuticas.

En terapia ocupacional los objetivos generalmente fueron en las áreas perceptual, coordinación motora e integración visomotora. Dentro de estas áreas se integraban objetivos relacionados: a) concentración, b) tolerancia a la tarea y c) el manejo de la frustración. El análisis de los expedientes mostró que algunas de las personas participantes trabajaban en niveles máximos de L4 y L5.

Algunos presentaron dificultades en el área de lectoescritura. Las terapeutas, con el fin de atender esta dificultad, integraron en sus terapias ejercicios que promovían el reconocimiento y discriminación de número, letras y la lectura de oraciones sencillas. Aquellos niños y niñas que trabajaron a un nivel de L2 y L3 en el área perceptual y visomotora tuvieron por lo general dificultades en lectoescritura; sin embargo, algunos, a partir de la terapia lograron identificar y discriminar letras adecuadamente.

En patología de habla-lenguaje los patólogos generalmente trabajaron objetivos relacionados con desarrollar destrezas en las siguientes áreas: a) lenguaje receptivo y expresivo dentro de distintas categorías semánticas (partes del cuerpo, partes de la casa, etc.), b) comprensión de lectura, c) secuencia, d) articulación a partir del uso adecuado de fonemas y e) pragmática. La mayoría de los niños y niñas que recibieron esta terapia trabajaban a un nivel de L3 en sus objetivos. Hubo dos que alcanzaron un progreso de L5, lo que significó una alta dentro de este tipo de terapia.

Una de las áreas de mayor dificultad que presentaron fue la comprensión de lectura. Los terapeutas de habla-lenguaje trabajaron esto a través de la lectura de cuentos y formulación de preguntas relacionadas con la narración, con el fin de proveerles habilidades para establecer secuencias y destrezas de compresión de lectura.

Identificamos verbalizaciones relacionadas con el progreso clínico de algunos en el área de lenguaje expresivo y receptivo en distintas categorías semánticas.

\section{Factores psicosociales}

Entre los factores psicosociales que favorecieron el progreso clínico de las personas participantes podemos destacar: a) la disponibilidad de los niños y niñas para asistir a las terapias, b) la participación e integración en la terapia de los padres, madres y personas encargadas, c) los acomodos razonables provistos por el personal escolar, d) el cambio de maestros y maestras, e) discusión de casos entre las terapeutas que brindan los servicios y f) buena comunicación entre los terapeutas y el personal escolar.

Identificamos factores que pueden exacerbar los síntomas de los niños, niñas y jóvenes afectando su progreso clínico. Estos fueron: a) la falta de integración de la madre, padre, o persona encargada en el proceso terapéutico, b) la limitación de estrategias adecuadas de disciplina de padres, madres, o personas encargadas, c) ausencias a las terapias, d) cambio de escuela, e) cambio de terapeuta, (f) retraso en la renovación de contrato de terapeutas, g) ausencias de maestros y maestras a la escuela, h) ausencia del padre en la vida del niño o niña e i) otras condiciones médicas de los niños y niñas.

\section{Fortalezas}

Las fortalezas identificadas en las personas participantes jugaron un papel importante en su progreso clínico. Tradicionalmente, el trabajo clínico en el área de salud promueve un acercamiento que parte de un modelo de déficit $y / 0$ de necesidades. Los proveedores y proveedoras de salud que parten de este modelo buscan aliviar los síntomas de la 
enfermedad, o condición, para lograr que las personas tengan un mejor funcionamiento en la sociedad. Esto redunda en que no se trabaje desde las fortalezas, habilidades, recursos y destrezas de las personas, como se recomienda desde el refortalecimiento, y las mismas no se integran en el proceso terapéutico ni para fomentar el progreso clínico. En este proyecto integramos en todas las disciplinas el enfoque de refortalecimiento $(18,19,20)$. La idea fue superar el modelo de déficit, en otras palabras, trabajar desde sus fortalezas y no desde sus deficiencias, fallas, faltas. Entre las fortalezas identificadas se encontraron: a) independencia, b) perseverancia, c) cooperación, d) liderazgo, e) tolerancia para realizar tareas, f) afectividad, g) motivación y h) manejo de emociones.

\section{DISCUSIÓN}

Es poco común que en proyectos de este tipo se desarrollen discusiones de casos interdisciplinarias, usualmente por razones de fronteras profesionales tradicionales como razones administrativas. Desde el refortalecimiento reconocemos la importancia de que el desarrollo de nuevos conocimientos está en comprender las redes y estimular nuevas conexiones (20). Por ello una de las primeras tareas asignadas a las terapeutas antes de iniciar su trabajo clínico fue realizar una presentación formal de cada una de las disciplinas a todo el equipo de trabajo. El resultado de este ejercicio fue muy ilustrativo; primero, porque requirió de las terapistas discusiones grupales (lo que dio paso a la clarificación desde donde ejercían su trabajo hasta llegar a ciertos acuerdos para poder describirlo a los demás); segundo, reconocer lo poco que sabíamos unas disciplinas de otras; y tercero, darnos cuenta de lo mucho que teníamos en común. Cuando miramos los resultados de los factores psicosociales no nos sorprendió constatar la poderosa influencia que tienen las madres en el progreso clínico de las personas participantes, toda vez que la figura de la madre es central en nuestra concepción de familia y usualmente está presente en casi todas las actividades relacionadas a sus hijos o hijas. Sin embargo, sí nos sorprendió ver como segundo factor protectivo, la figura del padre, quien en nuestra sociedad actual no cuenta con una fuerte valoración en el entramado familiar, y que en no pocas ocasiones, se recoge en el imaginario popular como "madre es una y padre es cualquiera". Este hecho nos debe llevar a repensar y recomendar una participación, aún más activa, de la figura paterna en la vida cotidiana de sus hijos(as) y en las terapias, especialmente, porque si tenemos en cuenta, la ausencia del padre en el hogar aparece como el tercer factor no protectivo.

Nos llamó especialmente la atención que de los principales factores no protectivos fueran las dificultades de lectoescritura de las personas participantes las que encabezarán la lista de factores psicosociales. Este caso nos ha llevado a repensar si las dificultades con estas destrezas están en la base de otros aspectos conflictivos en la vida de niños, niñas y jóvenes, tales como acoso escolar, dificultad en diversas áreas de conducta, comportamientos agresivos, fracaso escolar, depresión, entre otros.

Sobre el progreso escolar vale destacar, como señalamos desde el refortalecimiento, la importancia de comprender a la persona como un proceso colectivo en formación, que somos un "collage" de experiencias (20), toda vez, que el niño, niña y joven habitan simultáneamente varios contextos y es parte de redes de relaciones.

Concluimos, en primer lugar, que este proyecto cumplió con sus metas al lograr que más del $80 \%$ de los objetivos trazados en las terapias se cumplieran, así como más de la mitad de las personas participantes lograron, al mismo tiempo, tener progreso escolar. En segundo lugar, aunque en un $70 \%$ de los expedientes analizados se pudo constatar que hubo un cambio de terapeutas, podemos concluir que en la mayoría de los casos se logró progreso terapéutico en todas las disciplinas (factor indispensable para que ocurriera el progreso clínico). En tercer lugar, nos parece importante destacar que los factores que solemos llamar psicosociales tienen un peso considerable en facilitar u obstaculizar la terapia. En cuarto lugar, confirmamos la importancia de trabajar desde una perspectiva de refortalecimiento en lugar de una perspectiva de prevención para este tipo de proyecto. En quinto lugar, a través de la información cualitativa pudimos profundizar más sobre la cualidad del progreso clínico en áreas más específicas (manejo e identificación de emociones, autoestima, confianza en sí mismo, tolerancia, manejo de la frustración, lenguaje, comprensión de lectura, pragmática) y la dirección de dicho cambio (canalizar sentimientos, identificación de cualidades personales, mejora en relaciones interpersonales, mayor dominio de destrezas de atención, mayor calma, independencia, coordinación) lo que nos demuestra que la inversión en este tipo de proyecto de base comunitaria es necesaria e indispensable para mejorar la calidad de vida de esta población.

Otra conclusión importante es que debemos considerar para futuras investigaciones los aspectos relacionados con el género y su impacto en el desarrollo, diagnóstico y tratamiento de estas condiciones. Solo tomemos en consideración que casi el $100 \%$ de las terapeutas son mujeres y más del $66 \%$ de los participantes son varones. También resulta importante la participación de las terapeutas en las discusiones de caso, nos parece fundamental para atender las preocupaciones de los diversos sectores relacionados con las personas participantes ya que se convierte en un foro realmente 
interdisciplinario y sistémico para comprender mejor la realidad de esta población. Y la última conclusión es que pudimos observar que un $63 \%$ de las personas participantes tienen dos o más diagnósticos, lo que nos acerca a escenarios aún más complejos, que requieren una capacitación continua de nuestros profesionales, más allá de la formación en sus respectivas disciplinas, y desde enfoques como el refortalecimiento que superen el enfoque clínico tradicional.

\section{REFERENCIAS BIBLIOGRÁFICAS}

1 Rivera E, Fernández N, Torres A, Parrilla C. Análisis de la salud de Puerto Rico, Salud mental. San Juan; 2005. Disponible en: http://nesile.tripod. com/analisissaludmental.pdf

2 Bird H, Canino G, Rubio-Stipec M, Gould MS, Ribera J, Sesman $M$, et al. Estimates of the prevalence of childhood maladjustment in a community survey in Puerto Rico. Journal of the American Academy of Child and Adolescent Psychiatry. 1988; 45: 1120-1126.

3 Bird H, Yaget TJ, Staghezza B, Gould MS, Canino G, Rubio-Stipec M. Impairment in the epidemiological measurement of childhood psychopathology in the community. Journal of the American Academy of Child and Adolescent Psychiatry. 1990; 29: 796803.

4 Canino G, Bird H, Rubio-Stipec M, Bravo M, Martínez $R$, Sesman $M$, et al. The prevalence of specific psyquiatric disorders in Puerto Rico. Archives of General Psychiatry. 1987; 44: 727. 735.

5 Rosa Lydia Vélez vs. Awilda Aponte Roque: Caso $N^{\circ}$ KPE80-1738, Tribunal de Primera Instancia, Sala Superior de San Juan. (14 de febrero de 2002).

6 Canino G, Shrout PE, Rubio-Stipec M, Bird H, Bravo M, Ramírez R, et al. Archives of General Psychiatry. 2004; 61: 85-93.

7 Delgado N, Pacheco W, Reyes G, Rivera M. Pertinencia de un estudio de necesidades y recursos en un sistema de cuidado sensible a la diversidad de las familias latinas. Psychline: Journal of Hispanic American Psyquiatry. 2005; 4(6): 25-32.

8 Fundación Meniños. Evaluación de resultados en el Programa de Integración Familiar de Fundación Meniños. España; 2003.

9 Weiss $\mathrm{CH}$. Evaluation: Methods for studying programs and policies. New Jersey: Prentice Hall; 1998.

10 Rossi PH, Freeman HE. Evaluation: A systemic approach. California: Sage Publications; 1993.

11 Chelimsky E. Old patterns and new directions in program evaluation. En: Chelimsky E, compilador.
Program evaluation: Patterns and directions. Washington, D.C.: The American Society of Public Administration; 1985.

12 Shalock RL. Outcome-based evaluation. New York: Kluwer Academic/Plenum Publishers; 2001.

13 Noya B. Utilización terapéutica del yo. La relación terapéutica como base y refuerzo de la intervención. En: Polonio B, Durante P, Noya B. Conceptos fundamentales de terapia ocupacional. Madrid: Panamericana; 2001.

14 Arango Cammaert A, Moreno Franco M. Más allá de la relación terapéutica: Un recorrido histórico y teórico. Acta Colombiana de Psicología. 2009; 12(2): 133-145.

15 Munné F. La interacción social: Teorías y ámbitos. Barcelona: PPU; 1995.

16 Casas F. Infancia: perspectivas psicosociales. Argentina: Paidós; 1998.

17 Osorio M, Mejía L, Navarro J. Factores psicosociales que influyen en el éxito o fracaso del aprovechamiento escolar en la asignatura de física básica: Caso del plantel Ignacio Ramírez Calzada. Espacios Públicos. 2009; 12(26): 261 276.

18 Vázquez Rivera C. Refortalecimiento: Un debate con el empowerment. R. interam. Psicol. 2004; 38(1): 41-51.

19 Vázquez Rivera C, Escabí Montalvo A, Quiñones Sureda S, Pacheco Bou W. El refortalecimiento como una herramienta de trabajo comunitario: Reflexiones desde la comunidad. En: Zambrano A, Berroeta $\mathrm{H}$, editores. Teoría y práctica de la acción comunitaria: Aportes desde la Psicología Comunitaria. Chile: Ril Editores; 2012.

20 Vázquez Rivera C (prensa). Refortalecimiento y aprendizaje colaborativo: Desde una pedagogía de la pregunta. En: Huaire E, Elgier A, Maldonado $G$, editores. Psicología cognitiva y procesos de aprendizaje. Lima: UNE-EGV. 